\title{
Decline of Key Qualities in Medical Students: The what, why and how
}

\author{
Liu Jiaming
}

\begin{abstract}
Selection of medical students is becoming progressively more challenging with time. Medical schools struggle with selecting students who have key non-academic qualities that will enable them to be good doctors. However these qualities appear to be declining over the course of medical school. The qualities in decline, the causes for their decline and the suggested solutions to prevent their decline are discussed in this review article.
\end{abstract}

Key Words: Empathy, medical student selection, moral decline, medical education

\section{Introduction}

In addition to academic performance, the noncognitive component has begun to play a larger role in medical student selection, resulting in the replacement of traditional interviews with more rigorous methods such as the Multiple Mini Interview (MMI). The increasing importance of elucidating key personality traits in students before their entry into medical school can be attributed to recent studies that have shown erosion of certain qualities fundamental to being a doctor during medical school training.

\section{Key Qualities that are in Decline}

There are some key qualities that have been investigated in studies and identified to be in decline. They are: Ethical Skills and Moral Judgement, empathy and Cynicism and psychological issues.

\section{Ethical Skills and Moral Judgement}

Moral judgement competence encompasses the capacity to make decisions which are moral (i.e. based on internal principles) and to act in accordance with such judgements. It is measured as a construct that is similar to empathy.

In a cross sectional survey by Schillinger that examined medical students as well as psychology and economics students, it was found that moral judgement competency of economisc and psychology students improved

Dr. Liu Jiaming

NUHS Preventive Medicine Singapore considerably during their studies, while alarmingly, that of medical students worsened (Schillinger, 2006). Patenaude's study (Patenaude et al., 2003) looked at the requirements of professionalism and the expected qualities of medical staff. Using the French version of Kohlberg's Moral Judgement Interview, the authors assessed the progress in moral reasoning in a cohort of medical students over the first 3 years of their education. Results showed that there was a significant decline in moral character and ethical sensitivity over the 3 years. This was consistent with other studies performed in the United States that suggested a negative trend in the progress of ethical skills during medical training, manifesting in decreased ethical sensitivity, inhibited development in moral reasoning and serious erosion of ethical principles. There was no improvement of the situation even after completion of medical school (Hojat et al., 2009).

\section{Empathy}

Empathy is a multidimensional construct that encompasses both cognitive and affective components. The cognitive component of empathy is concerned with the ability to perceive another's point of view while being aware of one's effect on others. The affective component concerns one's vicarious emotional responses to the perceived emotional experiences of others. Empathy has long been acknowledged to be important for physician patient relationships, and can lead to personal growth, career satisfaction and optimal clinical outcomes (Hojat et al., 2009) (Chen et al., 2007). 
In a systematic review of studies by Neumann et al. (2011) a decline in empathy appeared to occur throughout both medical school and residency with a significant drop occurring once students entered the clinical phase of medical school. It was noted by Chen et al. (2007) that in spite of the decline in empathy, clinicians who chose to specialise in peopleorientated specialities tended to have higher empathy scores compared to those who chose to specialise in technology-orientated specialities, suggesting a form of natural preselection for career preferences amongst doctors. Female medical students were also found to score higher than their male counterparts. This was consistent for American, Mexican and Japanese medical students. The gender difference has been attributed to intrinsic as well as extrinsic factors, such as gender role expectations and interpersonal style in caring and socialization (Larson \& Yao, 2005; Hojat, 2007).

Interestingly, a study examining empathy levels amongst Japanese medical students did not show any observed decline in empathy (Kataoka et al., 2009).Conversely it showed that although Japanese medical students started medical school with lower empathy scores, their scores were more elevated than their American counterparts throughout the course of medical school. This was thought to be due to the inclusion of humanities and arts in their medical education curriculum, the limited amount of patient contact in the first four years of medical school and the use of only one national licensure exam at the end of sixth year as opposed to multiple steps of the USMLE as required of American students.

In contrast, a study done on Korean medical students and physicians showed that unlike the Japanese, Korean doctors scored lower on empathy than their American counterparts with a decrease in empathy score across the course of medical school (Dae et al., 2012). The former was attributed to the paternalistic role of Korean doctors in the doctor-patient relationship, while the latter was attributed to over reliance on biotechnology, insufficient patient contact time and high workload in hospitals.

\section{Cynicism and psychological issues}

Other qualities such as cynicism and atrophy of idealism were found to become more prevalent with time amongst medical students and residents, while commitment toward care for the medically underserved declined. Mood changes were also studied in conjunction with empathy levels. Not surprisingly, there was a high incidence of depression and anxiety among interns and residents with a peak at mid-internship and subsequent improvement during residency. Unfortunately, empathy scores also declined throughout internship only to remain low throughout residency (Bellini \& Shea, 2005).

\section{Proposed Reasons for Decline}

The reasons for this decline in key qualities are multifactorial and have been proposed by various authors to be a combination of the following: distress caused by curriculum issues, negative coping mechanisms, adjustment processes and social phenomenon.

\section{Distress caused by Curriculum Issues}

Much distress appeared to be contributed to by either hidden curriculums or the presence of a differentiated formal/ informal curriculum. Several aspects of a "hidden curriculum" that impacted negatively on empathy and ethical skills included mistreatment by superiors or mentors, lack of social support as students and residents, less time spent with families, high workload in combination with sleep deprivation, long working hours and inadequate relaxation time as well as vulnerability of idealism, enthusiasm and humanity in the face of clinical reality. Many students and residents also felt that the bureaucratic elements of medicine overshadowed the more human, empathic side. On the other hand, aspects of a "formal/informal curriculum" that were potential causes for ethical decline included short length of stay of patients resulting in fragmented patient-physician relationships, unsuitable learning environments such as unstructured studying, medical students being treated as immature human beings and inadequate role models (Fields et al., 2004).

\section{Negative Coping Mechanisms}

With increased patient responsibility and contact, students and residents may find that their initial ideals and expectations of the curative aspect of medicine do not match that of reality. As a result they react to the stress of this change in undesirable ways such as detached concern and decreased empathy.

\section{Process of Adjustment}

Some have argued that the decline in empathy levels is a normal process that healthcare professionals need to undergo to adjust to surroundings and demands of their particular field. However, it is pertinent to highlight that studies examining empathy levels in nursing 
students showed no decline in empathy across the course of their education and profession (Becker \& Sands, 1988) (Hojat et al., 2003).

\section{Social Phenomenon of $21^{\text {st }}$ century}

It is also possible that this ethical decline is part of a social phenomenon amongst the young. A study looking at more than 13,000 American college students identified a significant decline in self-perceived empathy from 1979 to 2009 with more recent college students demonstrating approximately $40 \%$ less empathy than students 20 years ago (Neumann et al., 2011). "Information flooding" is now common in education with increasing use of communication technology. This is thought to cause a degree of emotional anaesthesia in which perceptions of thoughts and emotions become increasingly suppressed. This in turn results in an inability to identify moral or ethical issues.

\section{Ways to Prevent Decline}

In order to prevent the decline in these key qualities, it is important to consider the factors that enable doctors to act in an ethical way. These can be categorised into factors that can be taught and factors that are innate to the individual. While certain personality traits that predispose to immoral behaviour such as narcissism, egotism and ethical disinterest are often intrinsic, the authors in a study on ethical assessment of medical school applicants felt that many unsophisticated ethical beliefs can be modified by medical education as students gain experience and broaden their knowledge base (Lowe et al., 2001).

\section{Stringent Selection of Medical Students}

Selection of students based on personality traits is difficult and the methodological issues involved in defining and testing ethical attributes are manifold. While narcissistic egotism and ethical disinterest in students are readily identifiable, the unethical behaviour of "authoritarian" type personalities is more difficult to ascertain. "Authoritarian" type personalities readily abide by authority but fail to stand out from unethical practices, leading to inappropriate behaviour later on in their professions. It is hopeful that the introduction of $\mathrm{MMI}$ and increase in emphasis on nonacademic components for medical school selection may aid selection on this front. However, it remains challenging.

There may also be a need to consider a change in the traditional mind-set of selecting students with heavily science-based backgrounds, as students with a humanities background prior to medical school have been found to have enhanced communication skills and a more humanistic approach to the patient. They were also found to have a heightened interest in fields that provide greater interpersonal connections between patient and physician. This more broad based premedical education may have a positive impact on the ethical and moral decline of students and future residents (Muller \& Kase, 2010).

\section{Continuous Education of Medical Students}

Ethical education should be part of the core curriculum in medical schools. Tutorials should look at improving interpersonal skills, using audio-visual media to analyse patient encounters and encouraging role playing. Students may be asked to shadow patients with chronic illnesses. In some medical schools, healthy medical students were made to experience hospitalisation in order to increase their empathy and understanding of the patient's experience. Some researchers have proposed that reading literature, watching movies and listening to music provide a rich source of insight about human emotions, pain and suffering. It also improves the capacity for forming empathic connections, and can aid the development of otherwise abstract clinical competencies such as moral reflection and accurate interpretation of ethical issues.

\section{Optimising the Environment for Medical Students and Residents}

The Balint method has been used by some residency programmes to improve the residency experience (Fields et al., 2004). This method involves small group meetings among residents for one or two hours every week or fortnight. The primary focus of these meetings is to allow for the discussion of behavioural, cognitive and emotional issues related to communication between patients, physicians and other personnel.

Minimising the "hidden curriculum" in the training of health professionals is essential. This will be challenging as the hidden culture of commercialism, financial preferences and authoritarian practice amongst seniors can be difficult to change.

One of the first steps towards creating a conducive learning environment is the development of appropriate role models for young doctors and students. It does not bode well that in a mailed survey of medical students at four different medical schools in Canada, $25 \%$ of second year students and 
$40 \%$ of seniors felt that their medical school faculty did not behave as humanistic physicians and teachers (Hojat, 2009).

Steps to improve work-life balance, decrease work load and increase time spent with family members will also go some way to improving the environment of medical students and residents.

\section{Conclusion}

Many studies have shown a moral, ethical and empathy decline amongst medical students and doctors. While there are some cultural, gender and specialty specific differences, the documented decline is disturbing. To prevent against this decline, efforts must be made to re-evaluate our selection criteria for medical schools, our undergraduate and postgraduate education and the hospital environment that our doctors and students become immersed in. By identifying and addressing the problems identified in these three key areas will we then be able to work effectively towards preventing this decline.

\section{References}

Becker, H. \& Sands, D. (1988) The relationship of empathy to clinical experience among male and female nursing students, Journal of Nursing Education, 27, pp. 198-203.

Bellini, L.M. \& Shea, J.A. (2005) Mood Change and Empathy Decline Persist During Three Years of Internal Medicine Training, Academic Medicine, 80, pp. 164-167.

Chen, D., Lew, R., Hershman, W. \& Orlander, J. (2007) A Cross-sectional Measurement of Medical Student Empathy, Journal of General Internal Medicine, 22, pp. 1434-1438.

Dae, H.S., Jong, S.H., Dong, H.L., Gonnella, J.S. \& Hojat, M. (2012) The Jefferson Scale of Physician Empathy: A preliminary psychometric study and group comparisons in Korean physicians, Medical Teacher, 34, pp. 464-468.

Fields, S.K., Hojat, M., Gonnella, J., Mangione, S., Kane, G. \& Magee, M. (2004) Comparisons of nurses and physicians on an operational measure of empathy, Journal of Educational Evaluation for Health Professions, 27, pp. 8094.

Hojat, M., Fields, S.K. \& Gonnella, J.S. (2003) Empathy: An NP/MD comparison, Nurse Practitioners, 28, pp. 45-47.

Hojat, M., Vergare, M.J., Mazwell, K., Brainard, G., Herrine, S.K., Isenberg, G.A., Veloski, J. \& Gonnella, J.S. (2009) The Devil is in the Third Year: A Longitudinal Study of Erosion of
Empathy in Medical School, Academic Medicine, 84, pp. 1182-1191.

Hojat, M. (2007) Empathy in Patient Care: Antecedents, Developments, Measurement and Outcomes, New York: NY Springer.

Hojat, M. (2009) Ten approaches for enhancing empathy in health and human services cultures, Journal of Health and Human Services Administration, 31, pp. 412-450.

Kataoka, H., Koide, N., Ochi, K., Hojat, M. \& Gonnella, J.S. (2009) Measurement of empathy among Japanese medical students: Psychometrics and scores differences by gender and level of medical education, Academic Medicine, 84, pp. 1192-1197.

Larson, E.B. \& Yao, X. (2005) Clinical empathy as emotional labor in the patient-physician relationship, Journal of the American Medical Association, 293, pp. 1100-1106.

Lowe, M., Kerridge, I., Bore, M., Munro, D. \& Powis D. (2001) Is it possible to assess the "ethics" of medical school applicants? Journal of Medical Ethics, 27, pp. 404-408.

Muller, D. \& Kase, N. (2010) Challenging Traditional Premedical Requirements as Predictors of Success in Medical School: The Mount Sinai School of Medicine Humanities and Medicine Program, Academic Medicine, 85, pp. 13781383.

Neumann, M., Edelhauser, F., Tauschel, D., Fischer, M., Wirtz, M., Woopen, C., Haramati, A. Scheffer, C. (2011) Empathy Decline and Its Reasons: A Systematic Review of Studies With Medical Students and Residents, Academic Medicine, 86, pp. 996-1009.

Patenaude, J., Niyonsenga, T. \& Fafard, D. (2003) Changes in students moral development during medical school: a cohort study, Canadian Medical Association Journal., 168, pp. 840-844.

Schillinger, M. (2006) Learning Environment and Moral Development: How University Education Fosters Moral Judgement Competence in Brazil and Two German Speaking Countries (Doctoral dissertation, Aachen: Shaker. 